\title{
Transverse mode coupling instability of colliding beams
}

\author{
S. White \\ Brookhaven National Laboratory, Upton, New York 11973, USA \\ X. Buffat \\ CERN, Geneva, Switzerland; EPFL, Lausanne, Switzerland \\ N. Mounet and T. Pieloni \\ CERN, Geneva, Switzerland \\ (Received 16 January 2014; published 9 April 2014; corrected 11 April 2014)
}

\begin{abstract}
In high brightness circular colliders, coherent and incoherent beam dynamics are dominated by beambeam interactions. It is generally assumed that the incoherent tune spread introduced by the beam-beam interactions is sufficiently large to cure any instabilities originating from impedance. However, as the two counterrotating beams interact they can give rise to coherent dipole modes and therefore modify the coherent beam dynamics and stability conditions. In this case, coherent beam-beam effects and impedance cannot be treated independently and their interplay should be taken into account in any realistic attempt to study the beam stability of colliding beams. Due to the complexity of these physics processes, numerical simulations become an important tool for the analysis of this system. Two approaches are proposed in this paper: a fully self-consistent multiparticle tracking including particle-in-cell Poisson solver for the beambeam interactions and a linearized model taking into account finite bunch length effects. To ensure the validity of the results a detailed benchmarking of these models was performed. It will be shown that under certain conditions coherent beam-beam dipole modes can couple with higher order headtail modes and lead to strong instabilities with characteristics similar to the classical transverse mode coupling instability originating from impedance alone. Possible cures for this instability are explored both for single bunch and multibunch interactions. Simulation results and experimental evidences of the existence of this instability at the LHC will be presented for the specific case of offset collisions.
\end{abstract}

DOI: 10.1103/PhysRevSTAB.17.041002

PACS numbers: 29.20.-c, 29.27.-a, 07.05.Tp

\section{INTRODUCTION}

Beam-beam effects are generally considered as the main limiting factor for luminosity performance in high brightness colliders. As they interact with each other, the two beams will couple, resulting in coherent oscillations. In the case of equal bunches and tunes these coherent oscillations can be described by two eigenmodes, corresponding to either in-phase or out-of-phase oscillations, respectively the $\sigma$ and $\pi$ modes. The tune shift between the $\sigma$ and $\pi$ modes is equal to $Y \cdot \xi$ where $Y$ is the Yokoya factor and $\xi$ the beam-beam parameter [1].

These coherent modes have been routinely observed in various colliders and are generally not self-excited [2-5]. However, the frequency of these modes may be well separated from the incoherent tune spread and consequently they do not profit from the large intrinsic Landau damping properties of the beam-beam interactions

Published by the American Physical Society under the terms of the Creative Commons Attribution 3.0 License. Further distribution of this work must maintain attribution to the author(s) and the published article's title, journal citation, and DOI.
[6]. Under external excitation, such as machine impedance, these modes could therefore become unstable. Assessing the stability of colliding beams therefore requires one to consider these two processes simultaneously and in a self-consistent way.

Past studies have shown that the combination of beambeam interactions and impedance could lead to coherent instabilities. However, these studies were either performed using a linearized model [7], i.e., not including Landau damping, or for very specific cases applied to the Tevatron [8]. During the 2012 proton run of the LHC, coherent instabilities of colliding beams were routinely observed [9], triggering a renewed interest to pursue these studies in a more general scope.

Analytical expressions describing the colliding beams system can become extremely complex when taking into account nonlinearities, the presence of multiple asymmetric collision points, and the contribution of multiple bunches to the coherent and incoherent beam dynamics. Numerical simulations are therefore required to study such complex configurations as relevant in the case of the LHC. Linearized model and multiparticle tracking simulations both offer advantages and disadvantages making them complementary tools to characterize and understand the 
coherent beam dynamics of this complex system. Both methods will therefore be used in the following. The motivation of this study being mainly driven by LHC observations, the LHC impedance model and beam parameters will be used, the numerical tools developed are however applicable to any configurations and the results should be qualitatively similar for other designs.

It should be noted that octupoles are extensively used during the whole LHC cycle to provide stability. The amplitude detuning introduced by the octupoles will modify the overall tune spread and when the beams are not colliding head-on their contribution, detrimental or not, can become important. Dedicated studies and analysis on the impact and role of the octupoles during the LHC operation cycle can be found in [10]. The contribution of octupoles will therefore not be further discussed but was taken into account when attempting to reproduce experimental data in Sec. VII.

In this paper we study the coherent modes arising from the interplay of beam-beam interactions and impedance and their stability dependence on various parameters such as chromaticity, interaction point (IP) optics functions, or transverse damper gain. The numerical tools used in this study, their implementation and the LHC impedance model are presented in Sec. II while benchmarking results are presented in Sec. III. The transverse mode coupling instability of colliding beams is introduced in Sec. IV and single bunch simulations as well as experimental observations are presented in Secs. V, VI, and VII. Finally, multibunch simulation results are discussed in Sec. VIII.

\section{MODELS}

Two models were developed to characterize the interplay of beam-beam and impedance: (i) a linearized model based on the circulant matrix approach introduced in [11], (ii) a fully self-consistent multiparticle tracking model. Single bunch effects were studied with the code BEAMBEAM3D [12] and multi-bunch effects with the code COMBI [13].

The circulant matrix model (CMM) allows us to compute the complex tune shift in the presence of $6 \mathrm{D}$ beam-beam interactions, impedance, chromaticity, and transverse damper. This approach is very fast and most appropriate for extensive parameter scans. However, the CMM is not a self-consistent approach, the elements of the longitudinal distribution are modeled as rigid objects and the beambeam force is linearized. As a consequence, the CMM is not capable of modeling nonlinear effects, in particular Landau damping which is most relevant when looking at beam stability issues, and does not allow us to account for the Yokoya factor leading to differences in the frequencies of the beam-beam modes with respect to theoretical expectations. An analytical derivation of a dispersion integral in LHC type of configurations promises to be a great challenge, in particular in the multibunch regime, i.e., in the presence of PACMAN effects. As a consequence, any attempt to predict measured stability threshold using this approach may fail. A numerical approach, by the means of self-consistent multiparticle tracking codes allows us to address these issues. While much more demanding in term of computing resources, tracking simulations are a necessary complement to the CMM. Such approach also allows us to treat any other nonlinear effects, such as transverse feedback imperfections or external noise. BEAMBEAM3D and COMBI are two similar implementations of such model, based on different multicore parallelization concepts. BEAMBEAM3D is optimized for single bunch studies allowing for full 6D beam-beam interactions while COMBI is designed to study multibunch configurations but is restricted to 4D beam-beam interactions. Depending on the case to be studied, it may be more appropriate to use one or the other.

These two approaches were implemented to allow for an arbitrary impedance model. This study was however motivated by LHC observations and the 2012 LHC impedance was used in all of the following simulations. Although the results would most likely be qualitatively similar, the impedance model certainly has an impact on stability thresholds and rise times. The impact of the impedance model goes beyond the scope of this study and will not be presented in this paper. For completeness, the LHC impedance model will therefore be described in this section together with the implementation of the circulant matrix and multiparticle tracking in our simulation codes.

\section{A. The circulant matrix model}

The circulant matrix approach was introduced in [11] and later extended to include single bunch head-on beambeam interactions in [7]. Here we shall describe how this method was extended to an arbitrary number of bunches and IPs including long-range interactions and bunch train structures.

Each bunch is represented as $N_{r}$ nested airbag distributions in the longitudinal plane, i.e., for a given airbag all the particles have the same synchrotron amplitude and are evenly distributed over the synchrotron phase resulting in a ring in the synchrotron phase space. Each of these rings is sliced into $N_{s}$ cells of equal weight characterized by their transverse dipole moments and synchrotron phase. It should be noted that arbitrary transverse distributions can be modeled by assigning different weights to the different rings. Considering only one transverse dimension, the synchro-betatron motion of a single ring is then described by a matrix $M$ consisting of $2 N_{s} \times 2 N_{s}$ elements. The synchro-betatron transport for a given arc is then given by

$$
M=C \otimes B
$$


where $\otimes$ stands for the Kronecker product. $B$ is the wellknown betatron transfer matrix

$$
B=\left(\begin{array}{cc}
\cos \mu_{\beta} & \beta \sin \mu_{\beta} \\
-\frac{1}{\beta} \sin \mu_{\beta} & \cos \mu_{\beta}
\end{array}\right) .
$$

In this example $\alpha$ was set to 0 and the $\beta$-functions at the IPs are assumed to be equal. $\mu_{\beta}$ is the betatron phase advance between two consecutive IPs. The synchrotron transport in each ring is described by the circulant matrix

$$
C=P_{N_{s}}^{N_{s} \mu_{s}},
$$

with $P_{N_{s}}$ the permutation matrix corresponding to a rotation of the cells by $1 / N_{s}$ turn in longitudinal phase space and $\mu_{s}$ the synchrotron phase advance.

The number of eigenvalues of the matrix $C$ is $N_{s}$ and represents the number of synchro-betatron modes to be computed. The number of slices has to be large enough in order to correctly compute the coupling between all the relevant modes and has to be derived from the convergence of the eigenvalues. It should be noted that the matrix $C$ is only describing the betatron motion in one transverse direction, the extension to the $2 \mathrm{D}$ case is rather straightforward and is left at the reader's discretion. The expansion of this matrix to two counterrotating beams consisting of $N_{b}$ bunches with $N_{r}$ rings is then given by

$$
M_{A}=I_{2} \otimes I_{N b} \otimes I_{N r} \otimes M,
$$

where $I_{n}$ is the identity matrix with $n \times n$ elements.

The linearized beam-beam parameter in the case of round beams is given by

$$
\xi=\frac{N_{p} r_{0}}{4 \pi \epsilon_{N}}
$$

where $N_{p}$ is the number of protons per bunch, $r_{0}$ is the classical particle radius, and $\epsilon_{N}$ is the normalized rms emittance. The angular kick received by a slice with transverse position $x_{1}$ from a slice with transverse position $x_{0}$ is given by

$$
\Delta x_{1}^{\prime}=\frac{2 \pi \xi}{N_{s} N_{r} \beta^{*}}\left(x_{1}-x_{0}\right),
$$

where $\beta^{*}$ is the beta function at the IP. The full 6D beambeam interaction is then described by a matrix $M_{b b}$ consisting of a succession of kicks and drifts taking into account the evolution of the $\beta$-function over the interaction region. This matrix is easily extended to offset collision by locally linearizing the beam-beam force and accordingly modifying the parameter $\xi$ in Eq. (6). The long-range interactions are lumped in two locations with phase advances of $\pm \frac{\pi}{2}$ with respect to the IP. The collision schedule at each IP is then properly taken into account

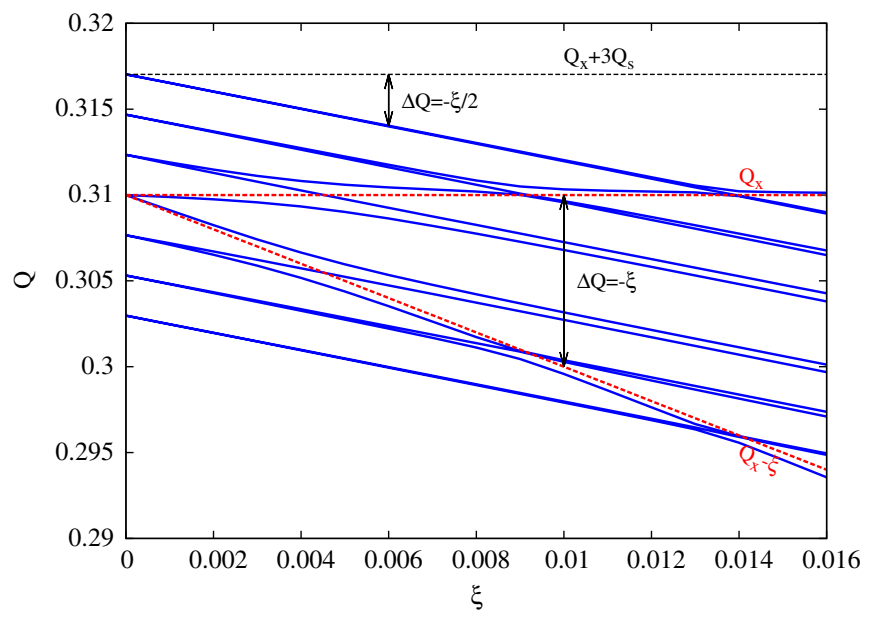

FIG. 1. Synchro-betatron modes as a function of the beambeam parameter for $Q^{\prime}=0.0$ and $\beta^{*} / \sigma_{s} \approx 1$. Impedance was not included in this case. The $\sigma$ and $\pi$ modes in the 4D case are shown in red. $Q_{x}$ is the unperturbed betatron tune and $Q_{s}$ is the synchrotron tune.

by computing the product of a succession of matrices describing the consecutive beam-beam interactions between the different bunches.

Figure 1 shows the tunes of the synchro-betatron modes up to the third sideband in the presence of a $6 \mathrm{D}$ beam-beam interaction for $N_{s}=7$ and $N_{r}=1$. In this case, the synchro-betatron coupling is rather important $\left(\beta^{*} / \sigma_{s} \approx 1\right.$, $\sigma_{s}$ is the rms bunch length). In addition to the tune shift introduced by the quadrupolar term of the beam-beam force, the synchrotron sidebands are deflected when their frequency approaches the coherent dipolar beam-beam modes indicating that the beam-beam force itself can excite headtail oscillations. This phenomenon is described in more detail in [7]. The imaginary part of the tune shifts of all modes is equal to zero: in the presence of beam-beam interactions only, the system is always stable.

The machine impedance is introduced by adding a kick to the $i$ th slice given by

$$
\Delta x_{1}^{\prime}=\mathcal{C} \sum_{j=1}^{i-1} W\left(s_{j}-s_{i}\right) x_{j}, \quad s_{j}>s_{i}
$$

where $\mathcal{C}$ is a constant depending on beam parameters and machine layout, $W(s)$ is the transverse dipolar wake function considered in the simulation, and $s_{i}$ is the location of the slice $i$. The same expression applies for coupledbunch impedance replacing $s_{j}-s_{i}$ by the distance between the bunches and performing the sum over all the leading bunches. Under the approximation that the long-range wake does not significantly vary along the bunch length the same kick is applied to all the slices within a bunch. This approximation is valid for the LHC as shown in Sec. II C. 
Finally, the one turn map is expressed as

$$
M_{1 T}=M_{b b 1} \cdot M_{A 1} \cdot M_{b b 2} \cdot M_{A 2} \ldots M_{b b n} \cdot M_{A n} \cdot M_{I},
$$

where $M_{I}$ is the matrix describing the impedance kicks. Other elements such as chromaticity or transverse damper can be described using the same matrix formalism allowing for realistic stability study of complex machines such as the LHC.

\section{B. Multiparticle tracking}

The two tracking codes used for this study, BEAMBEAM3D and COMBI, feature very similar implementations. The main differences reside in the field solver and the parallelization concepts. The field solvers are well documented in Refs. [12] and [14], respectively and will not be described in this paper. Both field solvers were extensively benchmarked and showed good agreement with theoretical expectations and experimental data. In BEAMBEAM3D, the parallelization is done on the bunch level allowing for fast computation of the 6D beam-beam kick. It is possible to track a limited number of bunches colliding in several IPs but the computing time scales linearly with the number of bunches and IPs. This code is most suited for the study of head-on effects and synchrobetatron coupling from the beam-beam interaction originating from crossing angle or hourglass effect. In COMBI, the parallelization is done on the beam level, i.e., each bunch is assigned to a given processor. This allows us to efficiently simulate complex collision patterns involving large number of bunches and interaction points. For computation efficiency the beam-beam kick is computed in 4D only, therefore neglecting synchro-betatron effects from the beam-beam interactions. In both codes the transport from one IP to the other is performed using the linear map from Eq. (2).

Both codes were extended to include self-consistent impedance calculation following similar implementation as the wakefields tracking code HEADTAIL [15]. The bunches are modeled as an ensemble of particles initialized with random Gaussian distributions in the 6 dimensions. After each turn the bunches are sliced longitudinally and the dipole moments of each of the slices as well as their weight is computed. The impedance kick for each of the slices is then derived from Eq. (7) both for intrabunch and coupled-bunch effects when relevant. Similarly to the CMM, transverse damper, chromaticity and in addition, nonlinear fields are available for stability studies.

\section{The LHC impedance model}

A detailed description of the LHC impedance model can be found in [16]. As most of the simulations presented in this paper make use of the 2012 LHC impedance model, a brief description of this model is provided in this section for completeness. The different contributions to the overall impedance can be separated into two main components for which analytical expressions can be derived: (i) The resistive wall impedance includes the 44 collimators, the beam screens ( $86 \%$ of the machine, in colaminated copper and stainless steel maintained below $20 \mathrm{~K}$ ) and the vacuum beam pipe (14\% of the machine, in copper at room temperature). The cross sections of each of these devices are properly taken into account in the impedance calculations. In particular, the half-gaps of the collimators are those measured during normal physics operation of the machine [17]. (ii) A broadband impedance is used to account for most of the smooth transitions located around the ring, as well as various geometric features such as pumping holes, beam position monitors, bellows, vacuum valves, and cavities [18].

In the numerical simulations, all the sources are lumped in one location taking into account the $\beta$-function at the source and at the observation point. This allows us to apply the impedance kick only once per turn.

Figure 2 shows the transverse dipolar wake functions at 4.0 TeV. The wake of both beams are almost equal, the horizontal plane is slightly stronger than the vertical plane. The quadrupolar wake, not shown on this figure, is also included in the model. One can see that the wake functions significantly vary over the bunch length, a large number of slices are therefore required for the numerical simulations to converge. On the other hand, the bunches are separated by at least $7.5 \mathrm{~m}$ in the LHC. The coupled-bunch wake does not vary significantly over the bunch length which allows us to apply the same kick to all the slices within a bunch.

Dedicated measurements were performed in the LHC to validate the impedance model during which tune shifts and instability rise times were measured as function of various parameters such as collimator aperture or bunch intensity [19]. Discrepancies of approximately a factor 2-3 were found between the measurements and the model which appears to underestimate the impedance.

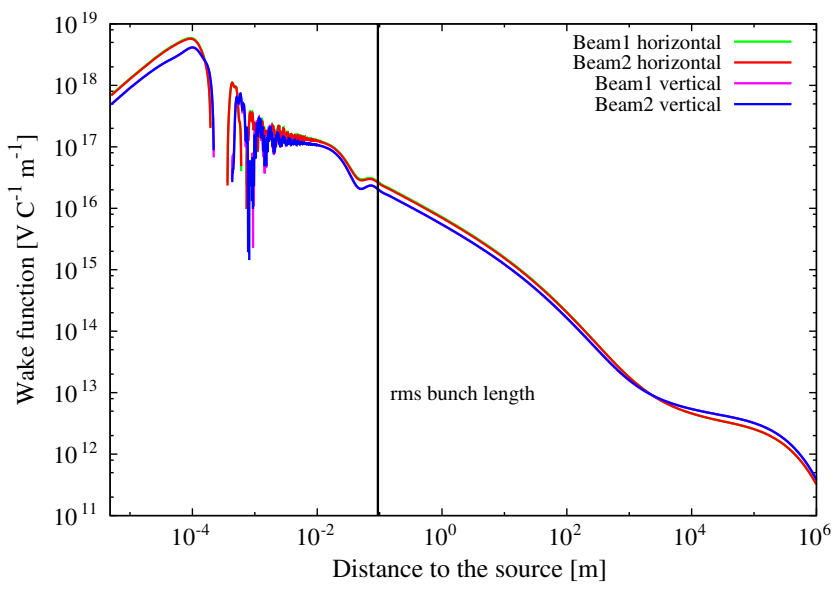

FIG. 2. Transverse dipolar wake functions at $4.0 \mathrm{TeV}$. Both axes are shown on logarithmic scales. 


\section{BENCHMARKING SIMULATIONS}

The interplay of beam-beam and impedance represents a very complex problem and only few references are available to validate our results. It is therefore necessary to carefully benchmark both the beam-beam and impedance modules of our simulations codes in order to gain confidence in the results.

\section{A. Beam-beam module}

The beam-beam modules of both tracking codes have already been benchmarked [12,14], we will therefore use them to validate the implementation of the CMM.

Figure 3 shows a comparison between tracking simulations and the CMM for $\beta^{*} / \sigma_{s} \approx 1$. The CMM was rescaled by the Yokoya factor to match the tracking results. Most of the synchro-betatron modes are not observed in the tracking simulations as they experience Landau damping due to the beam-beam tune spread. However, an excellent agreement is seen for the excited modes and one can see that the frequency of the modes is modified when the beam-beam coherent modes cross the first sidebands. The frequency components between the $\sigma$ and $\pi$ modes observed in the tracking correspond to the beam-beam tune spread.

Figure 4 illustrates the benchmarking simulations performed in the case of 4 bunches experiencing long-range interactions only in one IP. For long-range interactions $\beta / \sigma_{s} \gg 1$ and the beam-beam kick is computed in 4D only. The separation at the location of the long-range encounters is varied and we set $Q^{\prime}=10$ to increase the number of visible synchro-betatron modes. The agreement between the two models is excellent for large separations since the bunches see their counterpart as a whole. As the separation is reduced, the two models diverge due to the

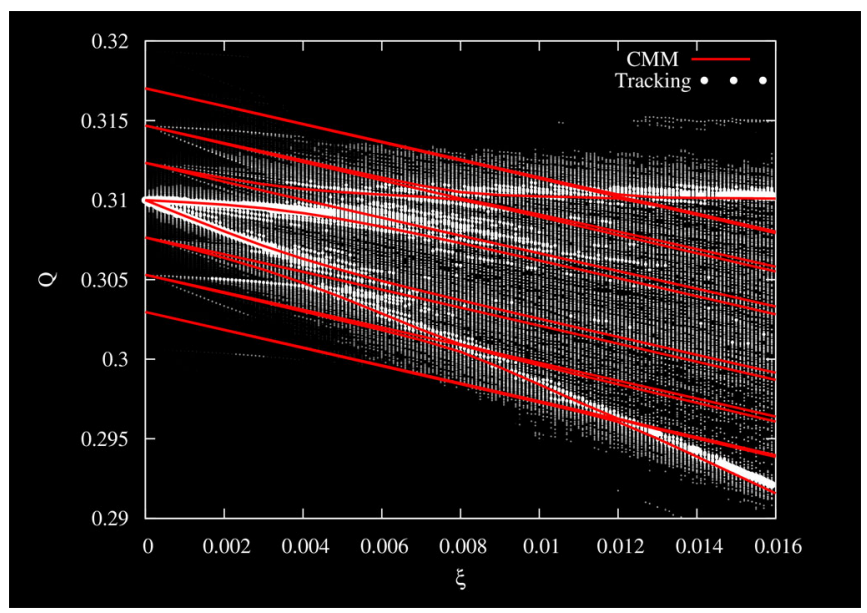

FIG. 3. Synchro-betatron modes as a function of the beambeam parameter for $Q^{\prime}=0.0$ and $\beta^{*} / \sigma_{s} \approx 1$. Impedance was not included in this case. The white dots are the results from the tracking and the red lines from the CMM.

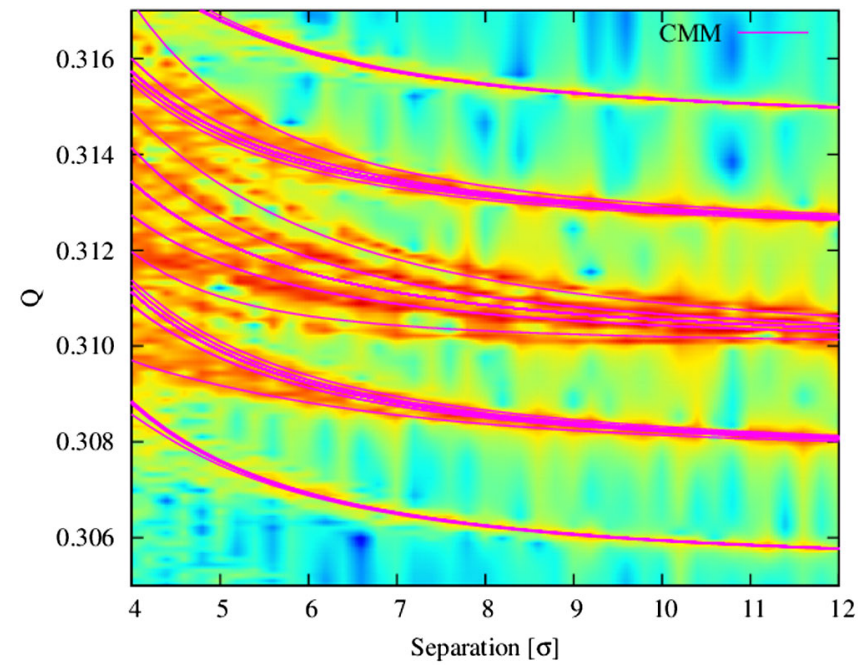

FIG. 4. Synchro-betatron modes as a function of separation at the location of the long-range encounters, with $Q^{\prime}=10.0 .4$ bunches per beam colliding in one IP are simulated. The colored spectrogram is the result from the tracking and the magenta lines from the CMM.

strong non-linear behavior of the beam-beam force at intermediate separations. This divergence is expected since the linearized model cannot account for the Yokoya factor.

In general, the CMM is in good agreement with selfconsistent tracking simulations. However, it does not allow us to account for the Yokoya factor and the tune shifts of the coherent beam-beam modes computed by the CMM are underestimated at small separations. This is not the case for long-range interactions for which the tune shift computed by the CMM is correct. Although the results from the CMM will be qualitatively correct, this feature will slightly bias their interpretation at small separations. Careful cross-checks with more realistic tracking simulations are therefore required to get the exact tune shifts.

\section{B. Impedance module}

The impedance module is new in both the tracking codes and the CMM. In order to validate its implementation we compared the results with the well-established wakefields tracking code HEADTAIL [15].

Figure 5 shows an intensity scan in the presence of impedance only and for $Q^{\prime}=0.0$ to check the consistency of the transverse mode coupling instability (TMCI) threshold [20] computed by our two models. The red lines are from the CMM and the colored spectrogram from multiparticle tracking. For the CMM simulation 5 radial modes (rings) and 100 azimuthal modes (slices per ring) were considered, the multiparticle tracking was performed with 200 slices and 500000 macroparticles. An excellent agreement is found between the two methods for which a threshold of approximately $4.1 \times 10^{11} \mathrm{p} /$ bunch was found. Similar simulations were performed with HEADTAIL for 


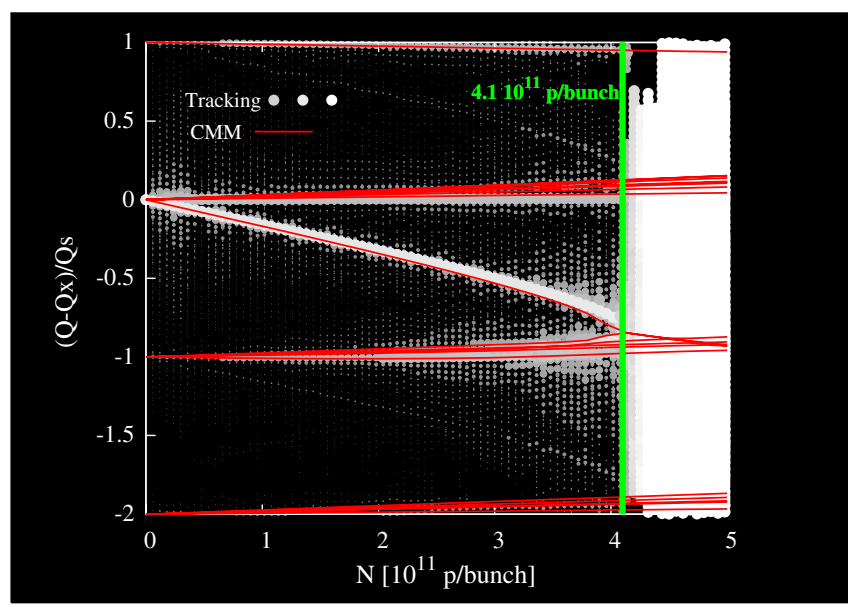

FIG. 5. Intensity scans at $Q^{\prime}=0.0$. The red lines are from the $\mathrm{CMM}$ and the colored spectrogram from multiparticle tracking.

which a TMCI threshold of $4.25 \times 10^{11} \mathrm{p} /$ bunch was found which is consistent within $5 \%$ with our implementation.

Figure 6 shows the growth rate of the most unstable mode for different chromaticity and damper gain settings. Only the unstable cases are shown. The results of our two models as well as the ones from HEADTAIL are shown. All codes agree very well up to a chromaticity of approximately 10. Above this value, some disagreements are observed between the tracking codes and the CMM for high damper gain. The growth rates for the tracking codes are obtained by fitting an exponential to the amplitude of the center of mass motion. In the presence of high damper gain and chromaticity these growth rates are very slow requiring us to track a very large number of turns and making it difficult to cleanly fit an exponential. Large fit errors can therefore explain these discrepancies.

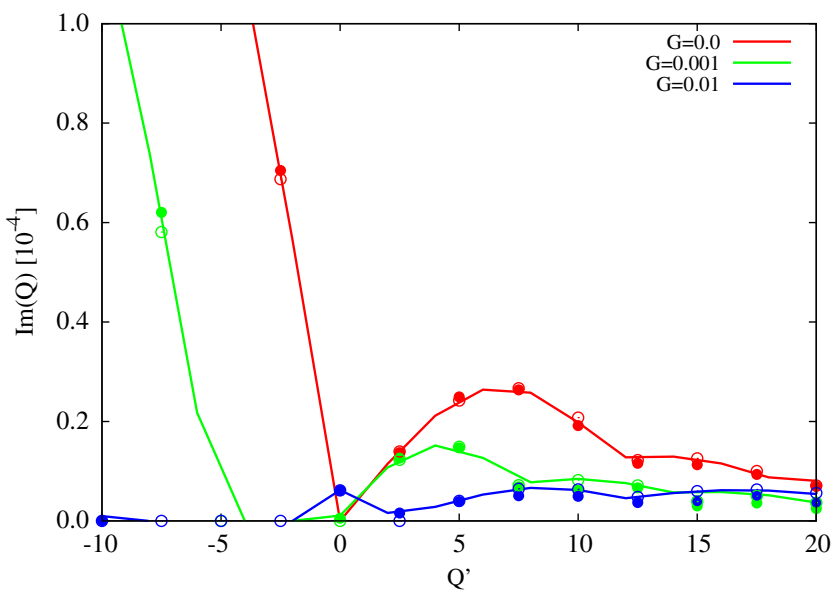

FIG. 6. Chromaticity scans with different transverse damper gains. The growth rate of the most unstable mode is shown. The lines are from the CMM, the plain dots from our tracking model, and the circles from HEADTAIL.
The impedance modules of both models were validated against an existing code. The results are in excellent agreement except for a few cases most likely related to numerical issues which are not a concern for further studies.

\section{MODE COUPLING INSTABILITY OF COLLIDING BEAMS}

We start by looking at the simple case of two bunches colliding head-on in one IP. The impedance model used in the following simulations is the one described in Sec. II C. The beam-beam interactions are computed with a full 6D model taking into account the synchro-betatron effects and eventual non-Gaussian transverse distributions. In order to estimate the beam stability for large number of beam parameters multiparticle tracking is performed over $10^{5}$ turns and each case is analyzed using an interpolated fast Fourier transform (FFT) algorithm. The beam stability of any given mode can then be assessed by looking at the amplitude of its corresponding spectral line.

Figures 7 and 8 show a scan in beam-beam parameter at constant impedance. As the beam-beam $\pi$ mode approaches the headtail mode $-1(\xi \approx 0.003)$, their frequencies become equal and they couple leading to strong instabilities with similar rise times and characteristics to the impedance driven TMCI. This is observed both in the tracking and circulant matrix model with comparable rise times.

As shown in Fig. 8, the circulant matrix model also indicates a coupling between the $\sigma$-mode and headtail mode +1 . This is not observed in the tracking simulations. However, the circulant matrix does not include nonlinear effects and hence, Landau damping. It was shown in [6] that in the presence of synchro-betatron coupling the coherent beam-beam modes could be damped by the sidebands of the incoherent tune spread. It is worth

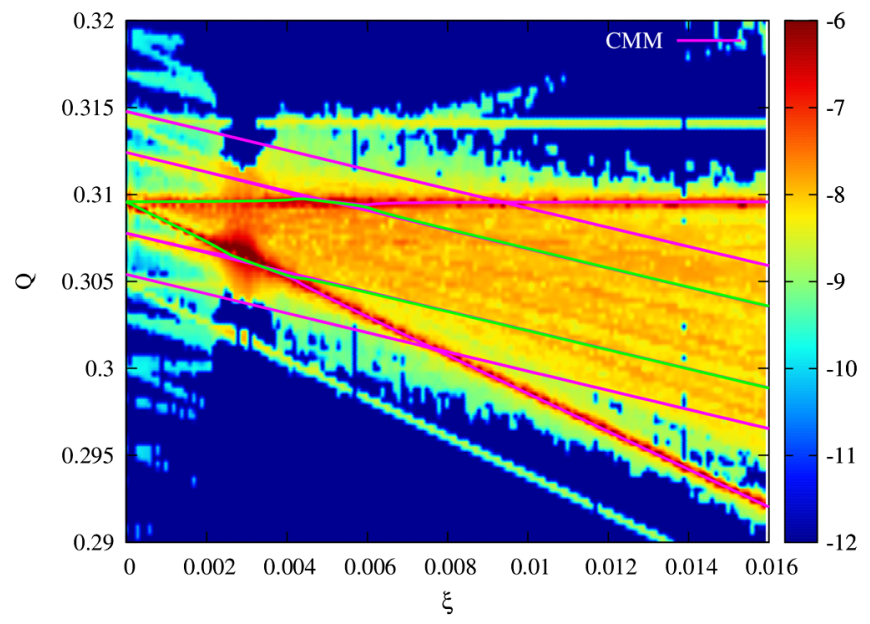

FIG. 7. Synchro-betatron modes as a function of the beambeam parameter for $Q^{\prime}=0.0$ and $\beta^{*} / \sigma_{s} \approx 90$. The colors correspond to the amplitude of the spectral line. Impedance effects were kept constant over the whole scan. The most unstable modes are shown in green. 


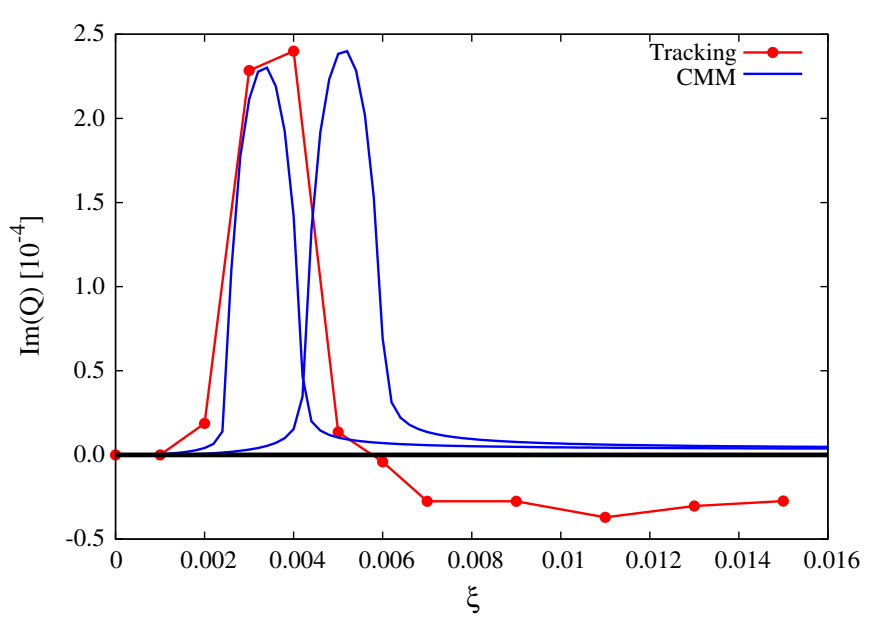

FIG. 8. Imaginary part of the tune shift of the most unstable modes as a function of the beam-beam parameter for $Q^{\prime}=0.0$ and $\beta^{*} / \sigma_{s} \approx 90$. Impedance effects were kept constant over the whole scan.

mentioning that beam-beam interactions can damp coherent oscillations not only through incoherent tune spread but also by a modification of the head-tail phase [6] or by changing the chromaticity itself [21] (which could also have detrimental effects).

As shown in Fig. 1 the frequency of the sidebands is approximately given by $Q_{0}+m Q_{s}-\xi / 2$ where $Q_{0}$ is the unperturbed tune, $Q_{s}$ is the synchrotron tune, $\xi / 2$ comes from the quadrupolar term of the beam-beam force and $m$ is the order of the sideband. The incoherent tune spread extends from $Q_{0}$ to $Q_{0}-\xi$ and its sidebands are centered around the frequencies $Q_{0}+m Q_{s}-\xi / 2$, one would therefore think that when a coherent beam-beam mode approaches a low order synchrotron sideband it would experience Landau damping. Nevertheless, the efficiency of the Landau damping depends on the particles frequency distribution around the frequency of the mode to be damped. The beam-beam tune spread is not uniform and most of the particles oscillate at frequencies lower than its central value while very few particles are located above. The coupling between the $\sigma$-mode and headtail mode +1 occurs with the mode +1 going down toward the $\sigma$-mode providing very efficient Landau damping, while it is the opposite for the coupling between the $\pi$ mode and headtail mode -1 for which Landau damping will therefore be very ineffective. This provides a possible explanation for discrepancies observed between the tracking and CMM results and why the coupling between the $\pi$ mode and headtail mode -1 appears to be much stronger in the case of head-on interactions.

\section{STABILIZING SINGLE BUNCH INSTABILITIES}

Chromaticity combined with tune spread, to provide Landau damping, is generally used to cure transverse instabilities. When available, as in the case of the LHC, a bunch-by-bunch transverse damper can also be used for this purpose. In order to get a better understanding of how these parameters affect the coherent beam dynamics and to provide guidance on which cases to study with the more time consuming multiparticle tracking, we start with the CMM.

Figure 9 shows the dependency of the imaginary part of the most unstable mode on the chromaticity and transverse damper gain as a function of the beam-beam parameter. For simplicity, we set the ratio $\beta^{*} / \sigma_{s}$ to 100 . Synchro-betatron coupling introduced by the beam-beam interaction can therefore be considered as negligible. The double peak structure characterizing the mode coupling instabilities shown in Fig. 8 is clearly observed. Operating at beambeam tune shifts where these instabilities occur is the most critical scenario for stability. It is seen that either high damper gain or chromaticity can help in mitigating these strong instabilities as the growth rate is significantly reduced. The case without beam-beam interactions is however not fully recovered and a combination of both may be more efficient.

Figure 10 shows the effects of the combination of the transverse damper gain and chromaticity with and without beam-beam interactions. Beam-beam interactions clearly degrade the situation for low damper gain or chromaticity which confirms the necessity to use an appropriate combination of both to mitigate the mode coupling instability. For high damper gain and chromaticity, which corresponds to the top right corner on the plots, the growth rates with or without beam-beam are very similar.

This is confirmed by looking at the projection for a gain of 0.01 (bottom right plot) where we can see that the curves almost converge when increasing the chromaticity. At high damper gain and chromaticity the stability of the beams is therefore mainly given by impedance. High damper gain or
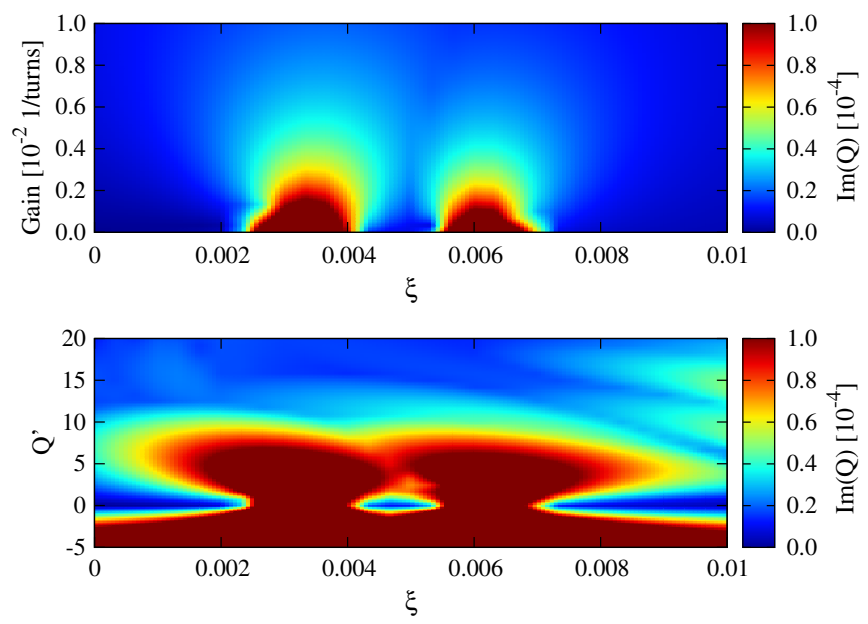

FIG. 9. Imaginary part of the tune shift of the most unstable mode (colors) computed by the CMM for different beam-beam parameters, transverse damper gain (top), and chromaticity (bottom). The ratio $\beta^{*} / \sigma_{s}$ is equal to 100 . 

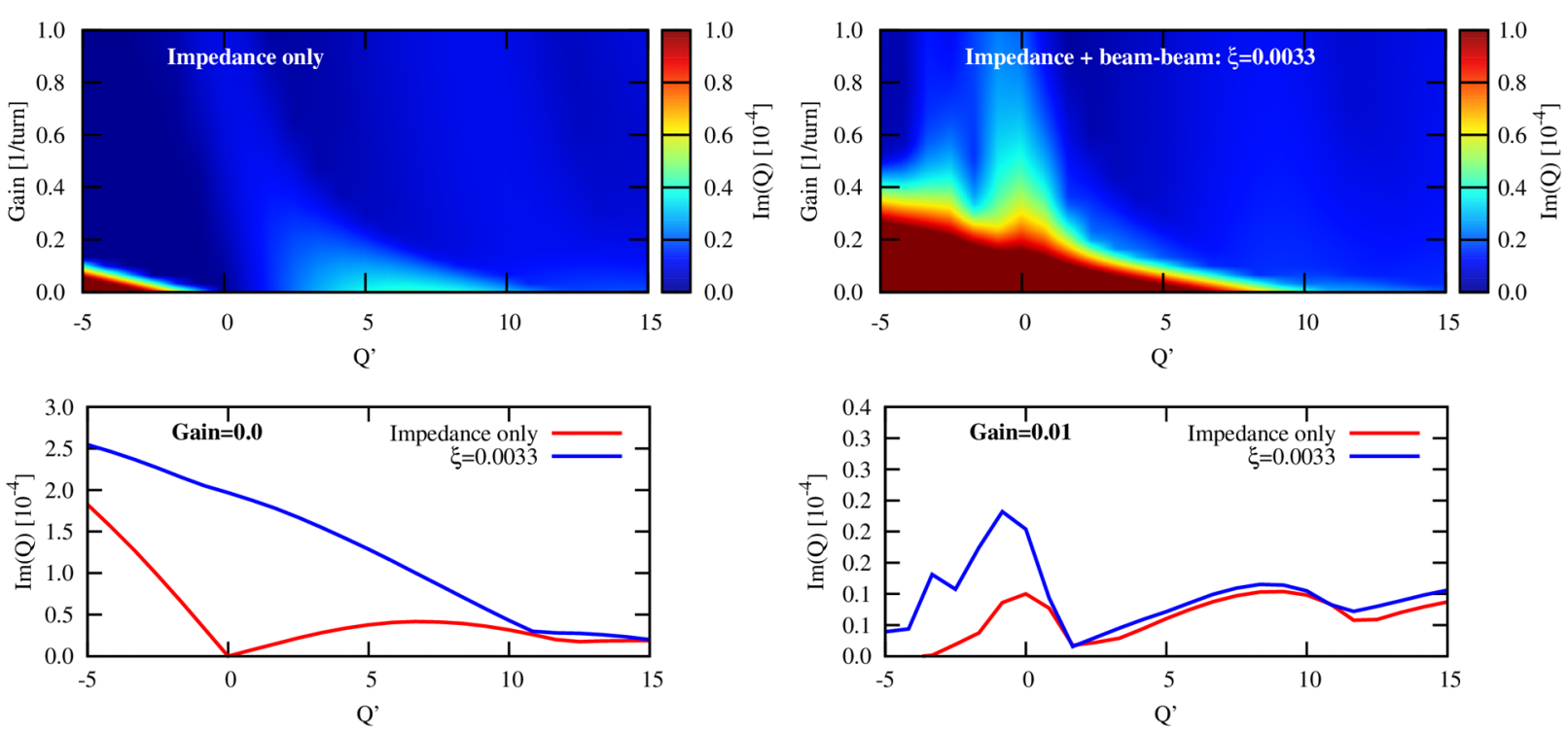

FIG. 10. Imaginary part of the tune shift of the most unstable mode (colors) computed by the CMM as a function of transverse damper gain and chromaticity. Several cases are illustrated. The case with impedance only is shown on the top left plot, the case corresponding to the mode coupling instability of the modes $\pi$ and -1 with $\beta^{*} / \sigma_{s}=100$ on the top right plot. Projections for a 0 transverse damper gain and a gain of 0.01 are shown on the two bottom plots.

chromaticity are however known to degrade beam lifetime, a careful optimization of these two parameters considering the overall machine performance is therefore recommended. For instance, the transverse damper appears to be much more efficient than chromaticity, one could therefore consider running at moderate chromaticity and high damper gain during the critical period of the operation cycle when the beams may become unstable. Alternatively keeping the beams colliding head-on, or quickly sweeping through the critical tune shifts when bringing the beams into collision would not only provide strong Landau damping due to the large beam-beam tune spread but also help avoid running into these strong mode coupling instabilities.

As mentioned in Sec. II the CMM does not include Landau damping which is an essential ingredient to assess beam stability. Although Fig. 9 provides some insight on possible mitigations, it is necessary to perform multiparticle tracking to get a more realistic and complete picture. In the following simulations the beam-beam tune spread is assumed to be the only source of Landau damping.

In order to illustrate the importance of Landau damping we consider two cases, one with head-on collision which profits from large Landau damping and one with a single long-range interaction at a separation of $10 \sigma$ for which Landau damping is very small. In both cases the beambeam tune shift was adjusted to be at the location of the mode coupling instability. The instability growth rate is obtained by fitting an exponential to the center of mass motion before emittance blowup, leading to a change in tune shift, is observed. The simulation results are shown in Fig. 11 where the head-on case is on the top plot and the long-range case in on the bottom plot. One can see that at zero gain and chromaticity the two cases are in good agreement together and with the calculation from the CMM shown in Fig. 10 within approximately 5-8\%. This is explained by the fact that even for head-on collision the $\pi$ mode is separated from the beam-beam tune spread by $Y-1$ where $Y$ is the Yokoya factor. In the absence of chromaticity it is therefore not subject to Landau damping.

As soon as the chromaticity is increased the head-on case starts to strongly diverge from the CMM or the long-range case, which remain in relatively good agreement. This
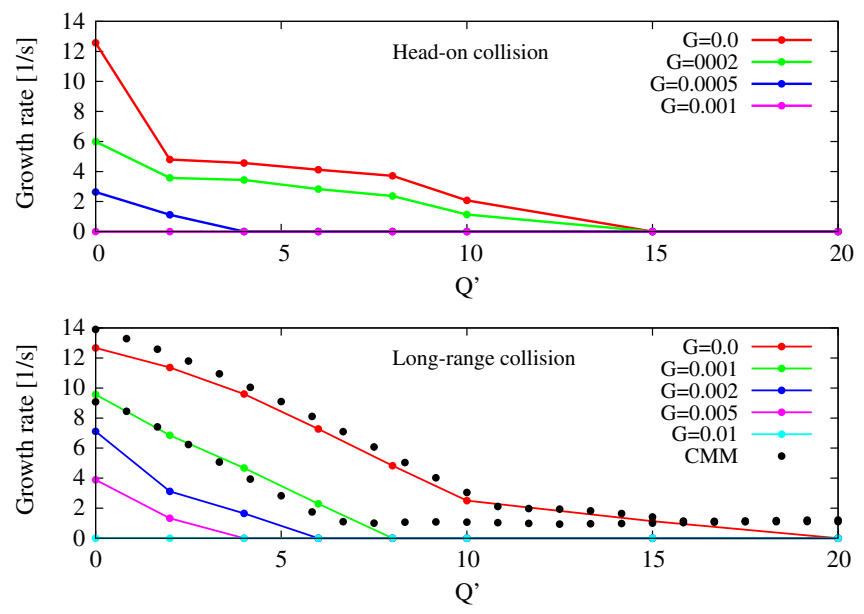

FIG. 11. Instability growth rate as function of damper gain and chromaticity for long-range (bottom) and head-on (top) collision. The beam-beam tune shift was adjusted in both cases to be at the location of the mode coupling instability. The wake is the same for both cases and the black dots are from CMM calculations. 
shows the stabilizing effect of the head-on beam-beam tune spread. A distinct behavior of long-range and head-on collision as function of the damper gain is also observed, i.e., the stabilizing damper gain is much higher for longrange interactions. It should be noted that the long-range case agrees well with the CMM calculation represented by the black dots for a few cases on the bottom plot. Again, Landau damping may play a role in this mechanism. As the damper will stabilize dipolar oscillations only it is fairly possible that other higher-order headtail modes are consequently excited. These would be damped by the large head-on beam-beam tune spread while the small tune spread introduced by long-range interactions is not sufficient.

The CMM results are confirmed by tracking simulation as it is seen that both chromaticity and transverse damper can mitigate the transverse mode coupling instability. Similarly, the transverse damper appears to be very efficient, while very high chromaticity would be required in the absence of transverse damper.

\section{STABILITY WITH OFFSET COLLISIONS}

Colliding with transverse offsets changes the frequency of the beam-beam coherent modes, modifies the beambeam tune spread and thus, its stabilizing properties. Offset collisions can occur while bringing the beams into collision, in the first moments of a physics store before the luminosity is optimized or when leveling the luminosity as was routinely done at the LHC in 2012 [22]. Figure 12 illustrates a scan in separation including coherent beambeam effects and impedance at zero chromaticity and damper gain. The mode coupling instability is observed when either the $\pi$ mode overlaps the headtail mode -1 or
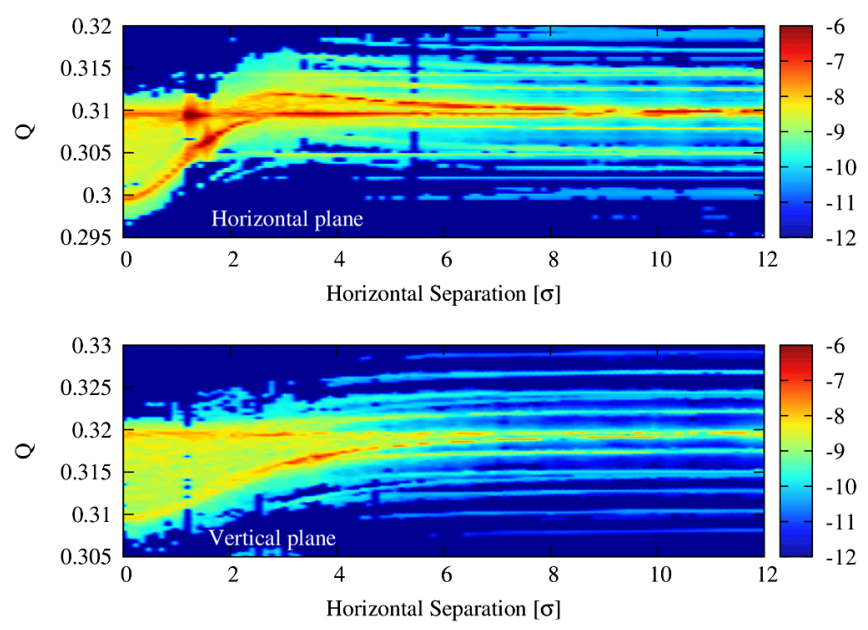

FIG. 12. Synchro-betatron modes from tracking simulations as a function of the transverse separation for $Q^{\prime}=0.0$ and $\beta^{*} / \sigma_{s} \approx 90$. The colors correspond to the amplitude of the spectral line. Impedance effects and beam-beam parameters were set to be constant over the whole scan. the $\sigma$-mode overlaps the headtail mode +1 at separations between 1.0 and $2.0 \sigma$. This is also the location at which the beam-beam tune spread reaches a minimum [23] explaining the differences with respect to head-on collisions.

\section{EXPERIMENTAL OBSERVATIONS AT THE LHC}

In order to confirm our simulation results a short experiment was performed at the end of a fill for which there were only two bunches per beam. Applying a transverse offset is an efficient way to vary the beam-beam parameter without having to reinject bunches of different intensities. Initially, the bunches are colliding in two IPs with a transverse offset of approximately $6.0 \sigma$. The offset is applied in the horizontal plane in one of the two IPs and in the vertical plane in the other IP. The chromaticity was measured at the beginning of the experiment and was found to be of the order of $8.0 \pm 2.0$ units in the horizontal plane and $4.0 \pm 1.5$ units in the vertical plane for both beams. The bunch intensity was approximately $1.1 \pm$ $0.05 \times 10^{11} \mathrm{p} /$ bunch and the beam emittances $\epsilon_{x} \approx$ $2.15 \mu \mathrm{m}$ and $\epsilon_{y} \approx 1.6 \mu \mathrm{m}$ for Beam $1, \epsilon_{x} \approx 2.25 \mu \mathrm{m}$ and $\epsilon_{y} \approx 1.9 \mu \mathrm{m}$ for Beam 2. The synchrotron tune $Q_{s}$ equals $2.3 \times 10^{-3} \cdot t=0 . t=0$ corresponds to the time at which the bunches were brought into head-on collision at the vertically separated IP. The other IP is kept separated at $6.0 \sigma$ in the horizontal plane during the whole experiment. The vertical separation is then increased in steps and at each step the transverse damper is turned OFF while the beam centroid motion is observed as a measure of stability.

Figure 13 shows a spectrogram of the vertical plane of Beam 1 over the duration of the whole experiment. When separated by $6.0 \sigma$, the vertical plane of beam 1 was found to be unstable without transverse damper for an octupole current up to $480 \mathrm{~A}$. These single beam instabilities can be observed before $t=0$ when the octupole current was raised in steps in an attempt to stabilize the beam without

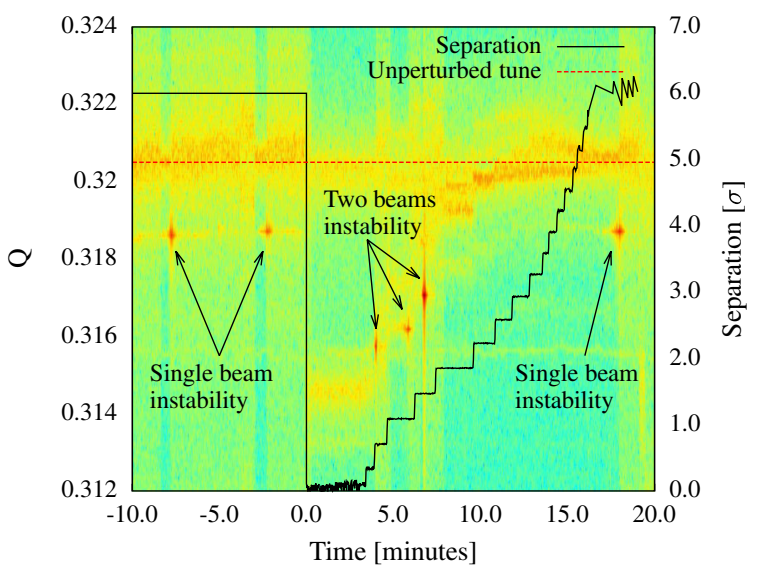

FIG. 13. Spectrogram from turn-by-turn data for the vertical plane of beam 1 . The separation is derived from luminosity data. 

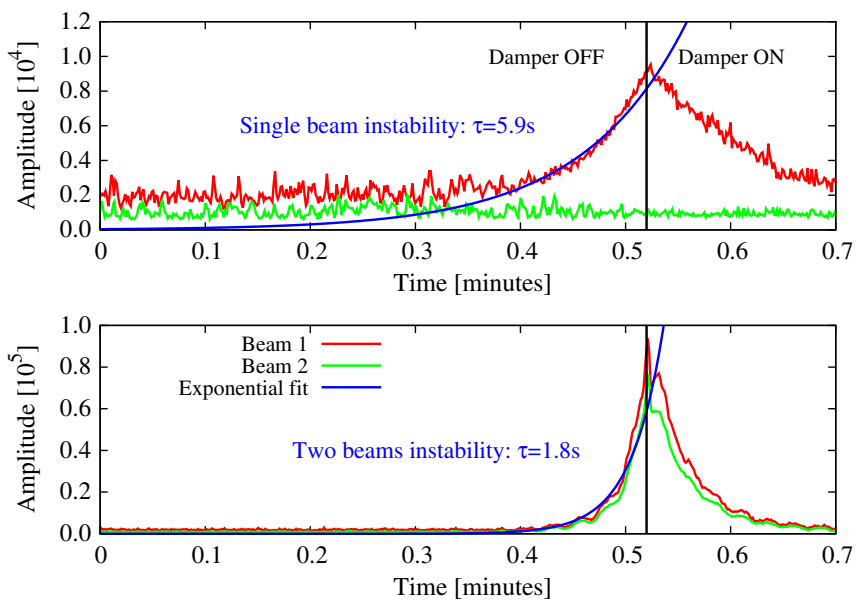

FIG. 14. Measured oscillation amplitude in the vertical plane of both beams and exponential fit. An example of the single beam instabilities and two beams instabilities are shown on the top and bottom plots, respectively. The time at which the damper was turned $\mathrm{ON}$ is marked by the vertical black line.

transverse damper and at the end of the separation scan when the separation is back to $6.0 \sigma$. The measured rise time for these single beam instabilities was estimated to $5.9 \mathrm{~s}$ as seen on the top plot of Fig. 14. In this case the frequency of the unstable mode appears to correspond to the first lower synchrotron sideband (headtail mode -1 ).

Bringing the beams into collision in one of the two IPs, at $t=0$, provides stability even in the absence of transverse damper. This was expected from the large beam-beam tune spread and the absence of mode coupling instabilities at $\xi \gg Q_{s}$. As the vertical separation is increased in steps we observed the appearance of instabilities for intermediate separations between $0.7 \sigma$ and $1.4 \sigma$ corresponding to separation at which the $\pi$ mode overlaps the first synchrotron sideband and the beam-beam tune spread reaches a minimum. These instabilities have quite different characteristics than the ones observed at large separation. They appear to involve both beams at the same time and their rise time was measured to be approximately $1.8 \mathrm{~s}$, as seen on the bottom plot of Fig. 14, which is approximately a factor 3 faster than the single beam instabilities. Finally, the frequency of the unstable mode is different and is consistent with the beam-beam $\pi$ mode.

It should be noted that all instabilities in this experiment were effectively damped by the transverse damper. This shows that a rigid bunch damper is not only effective against instabilities involving beam-beam coherent dipole modes but also instabilities driven by higher order headtail modes (in this case mode -1 ). This is in accordance with predictions of both the CMM and tracking simulations.

These observations could be consistent with the mode coupling instability shown in Fig. 12. However, the separation at which the instability is observed also corresponds to the configuration at which the beam-beam tune spread, and hence Landau damping, reaches a minimum. For this reason, one cannot exclude the possibility of a single beam instability due to the reduction of Landau damping which is then transferred to the other beam via the beam-beam force. The lack of diagnostics capable of correlating the turn by turn position of the two beams prevents a distinction between these two effects. Although the distinct characteristics of the single beam and two beams instabilities observed during this experiment appear to confirm the existence of the mode coupling instability involving coherent beam-beam modes, these measurements cannot be considered as fully conclusive. Numerical simulations are therefore required to be able to disentangle coherent and incoherent effects in this specific configuration and should provide the necessary information to conclude on the role played by the coherent beambeam modes.

As mentioned in Sec. II C the impedance model tends to underestimate the tune shifts by a factor $2-3$, to account for these observations the wakefields are multiplied by a factor 2. Large uncertainties on the chromaticity and emittance measurements, effective nonlinear fields and the LHC impedance model itself make it difficult to reproduce the experimental results, we will therefore empirically determine the parameters from simulations to match the observations made for separated beams. These parameters will then be used in further simulations. As pointed out in [21], beam-beam interactions themselves can contribute to a significant change in chromaticity while bringing the beams into collision as was done in the experiment. This effect was estimated to be of the order of 1.0 unit at most which is comparable to the accuracy on the chromaticity measurement and hence should not affect the interpretation of the results.

The experimental data for separated beams do not show any sign of coupling through the beam-beam force as only one of the two beams was observed to be unstable. This could be explained by the asymmetry between the beams in size and intensity which could cancel the coherent beambeam modes [6]. We therefore start by looking at the case with impedance combined with the tune spread from the octupoles and the beam-beam interactions.

Figure 15 shows the simulated instability growth rate as a function of chromaticity and octupole current. The first thing to note is that even with twice the wakefunctions the model predicts stability at any positive chromaticity for the measured octupole currents. Nevertheless, a reduction of approximately $10-20 \%$ of the detuning would allow us to observe instabilities with growth rate consistent with observations over part of the measured chromaticity range. Such a difference could easily be accounted for by field errors or uncertainty on the emittance measurement. In addition, the measured emittance in the unstable plane was approximately $1.6 \mu \mathrm{m}$. As a consequence, one can expect the effective vertical detuning due to the octupoles to be 


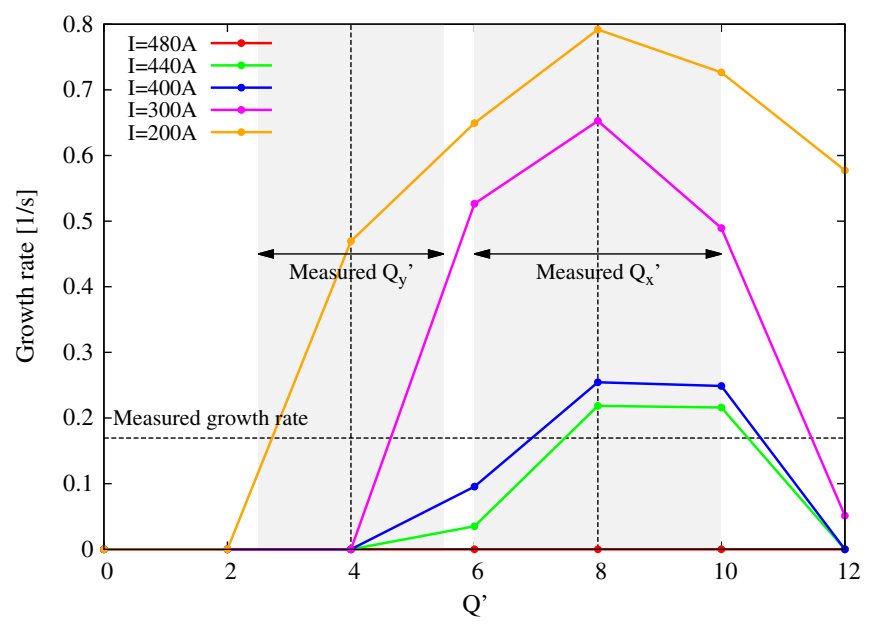

FIG. 15. Simulated instability growth rate in the presence of impedance and beam-beam spread for different octupole currents as a function of chromaticity. For these simulations the emittance was set to $2.0 \mu \mathrm{m}$. The gray areas represent the statistical uncertainty on the chromaticity measurements.

smaller than the simulated one, and hence the beam to be more unstable in this given plane.

The main inconsistency with data resides in the fact that with the measured chromaticities, the horizontal plane should always be more unstable than the vertical plane which is the opposite as what was observed experimentally. Such a feature cannot be explained by the impedance model nor by beam-beam interactions, which have the same impact in both planes (assuming equal emittances). The chromaticity measurement is achieved by applying a momentum offset which results in a change of orbit. Even though the beams were separated by $6 \sigma$, the beambeam force cannot be neglected and may have biased the chromaticity measurements due to its dependency on transverse separation. In order to match the experimental data the chromaticities of the horizontal and vertical planes were swapped, resulting in the horizontal plane to be more unstable than the vertical plane.

Figure 16 shows the instability growth rate of the vertical plane as a function of vertical separation. The results were obtained using a full 6D strong-strong simulations including nonlinear rf, crossing angle, and octupoles with bunches colliding in two IPs. The parameters used for these simulations correspond to the measured ones except for the chromaticity. The weak-strong simulations were performed with the parameters of Beam 1. A strong instability involving the 2 beams is observed with the strong-strong simulations for separations of 1.0 and $1.5 \sigma$ (the separation steps are $0.5 \sigma$ ). The simulated rise time is twice larger than the measured one which is within the uncertainty on the wake, nonlinearities, emittances, or bunch intensity. The frequency of the unstable mode is consistent with the beam-beam $\pi$ mode. A weaker instability is observed for separations larger than $5.0 \sigma$. This

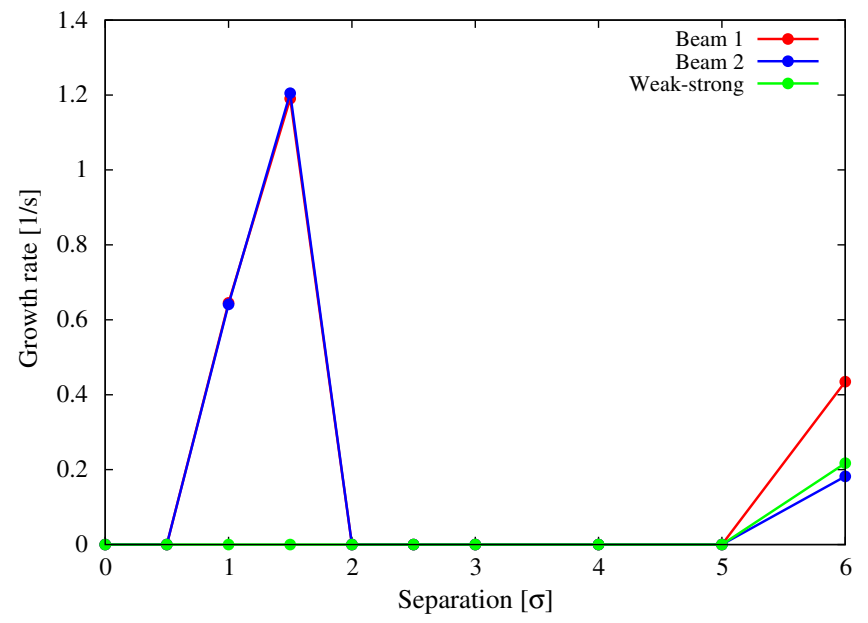

FIG. 16. Simulated instability growth rate in the vertical plane as a function of separation. The horizontal plane was stable for all separations.

instability appears to affect also both beams but Beam 1 is the most unstable. The frequency of the unstable mode is consistent with a headtail mode -1 in this case. In the weak strong simulations, for which there are no coherent beambeam modes, no instabilities are observed for intermediate separations. Although the rise-times are significantly different with respect to the ones observed in the machine the results qualitatively agree with the measurements: a strong instability is observed at intermediate separation with evidence of coupling between the beams while a much weaker instability is observed at large separation for which the coupling between beams appears to be much weaker. A small tune split of few $1.0 \times 10^{-4}$ would fully decouple the two beams. The weak-strong simulations do not feature the fast instability at intermediate separations providing a strong argument toward the observation of a mode coupling instability in the experimental data.

\section{MULTIBUNCH SIMULATIONS RESULTS}

In the LHC, for $25 \mathrm{~ns}$ bunch spacing, individual bunches can experience up to 32 long-range interactions per IP (this number goes down by a factor 2 for $50 \mathrm{~ns}$ bunch spacing), where the separation between the two beams is approximately $10 \sigma$. In some cases, the accumulated tune shifts from these interactions can become larger than the synchrotron frequency, potentially leading to mode coupling instabilities. In our model, each interaction region is modeled as a series of long-range interactions with constant separation located at phase advances of $\pm \pi / 2$ from the IP. Once in collision, the beam dynamics is dominated by the head-on interactions which was discussed in Sec. V. We will therefore concentrate on the case with long-range interactions only which corresponds to the LHC squeeze (period over which the $\beta$ function at the IP is reduced to enhance luminosity). Over this period the separation at the 

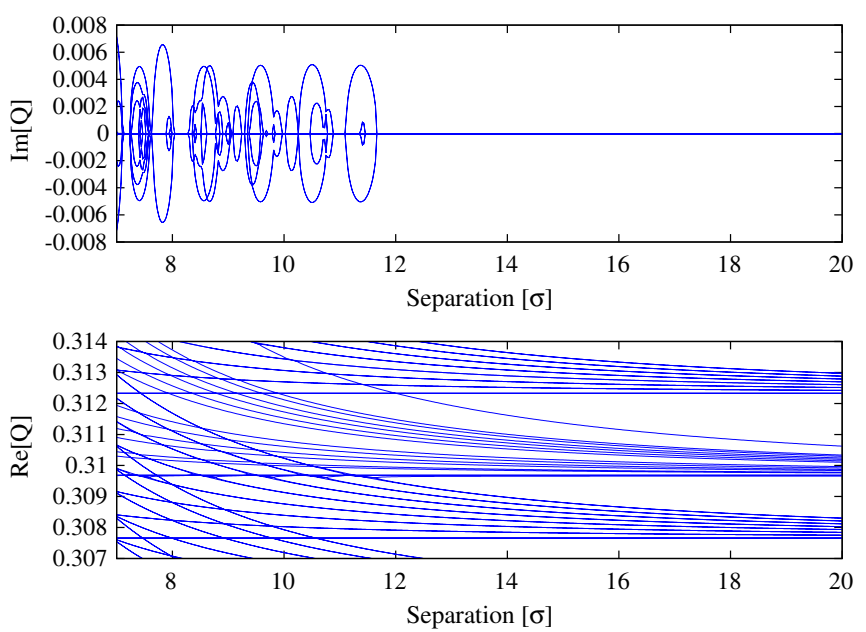

FIG. 17. Real (bottom) and imaginary (top) part of the coherent tunes of the modes obtained with the CMM for two trains of 8 bunches colliding in one IP with $Q^{\prime}=0$ and no transverse feedback. The PACMAN effects are enhanced by allowing collisions only on one side of the IP.

IP is of the order of $40 \sigma$. This additional long-range encounter is therefore neglected in the following analysis. At the locations of the long-range encounters $\beta / \sigma_{s} \gg 1$ and the beam-beam interaction can be reduced to the $4 \mathrm{D}$ case. As shown in Sec. III a direct comparison of the tune shifts obtained with the CMM is possible for separations of the order of $10 \sigma$ which makes it a very valuable tool to study the case of long-range interactions.

Due to their train structure, the LHC beams suffer the so-called PACMAN effects: bunches along the trains will experience different number of long-range encounters and hence have different tune shifts leading to a large variety of coherent modes with nearby frequencies.

Figure 17 illustrates the impact of PACMAN effects for a simplified case. Two trains of 8 bunches are colliding in a single IP, the PACMAN effects are artificially enhanced by allowing collisions only on one side of the IP. Each bunch therefore experiences up to 7 long-range interactions. Even for this simplified case a large number of mode coupling instabilities are observed involving various modes. As the separation is reduced, and hence the beam-beam tune shift is increased, the probability for two modes to overlap becomes higher and in some cases multiple mode coupling instabilities can occur simultaneously.

\section{A. Stabilization techniques}

In this analysis, we consider two beams composed of a single train of 16 bunches colliding in one IP. These bunches experience from 8 to 16 long-range interactions depending on their position in the train. The imaginary part of the tune of the most unstable mode, with $Q^{\prime}=0$ and no transverse feedback, is shown on Fig. 18. Two peaks are observed for which the imaginary part of the tune goes up

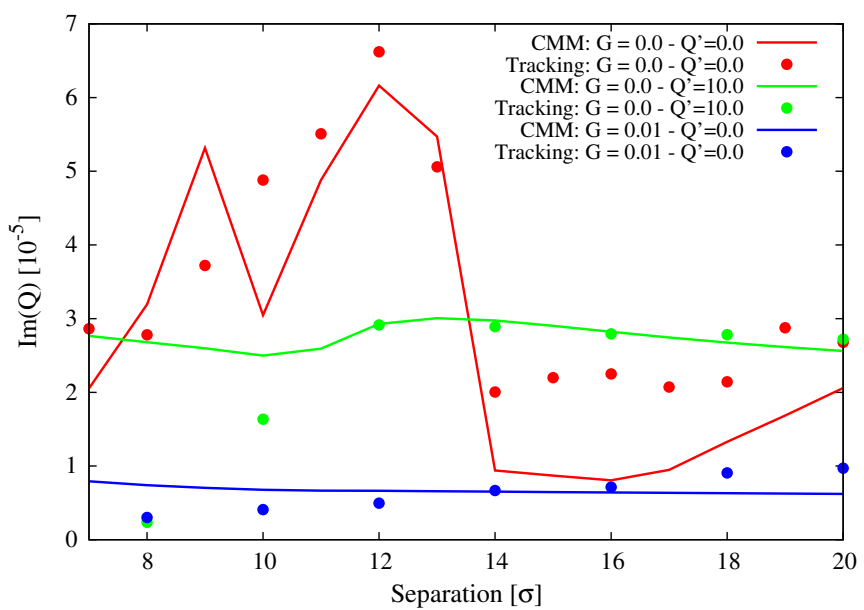

FIG. 18. Imaginary part of the tune of the most unstable mode obtained with the CMM (lines) and tracking (dots) for 2 trains of 16 bunches colliding in one IP as a function of the separation at the long range encounters for different chromaticity and damper gain.

to high values. They correspond to distinct mode coupling instabilities. The peak at separations between 11 and $13 \sigma$ corresponds to the strongest instability and involves the beam-beam $\sigma$-mode and the headtail mode -1 .

Figure 19 shows the imaginary part of the tune of the most unstable mode derived with the CMM as a function of both chromaticity and damper gain. The separation was set to $13 \sigma$ for which strong mode coupling instabilities are observed. Above 500 turns damping time or a chromaticity of 10 a significant decrease of the growth rate is observed indicating a mitigation of the mode coupling instability.

As a result of PACMAN effects, the beam-beam tune spread is different for each bunch making it very difficult to analytically introduce Landau damping to the CMM

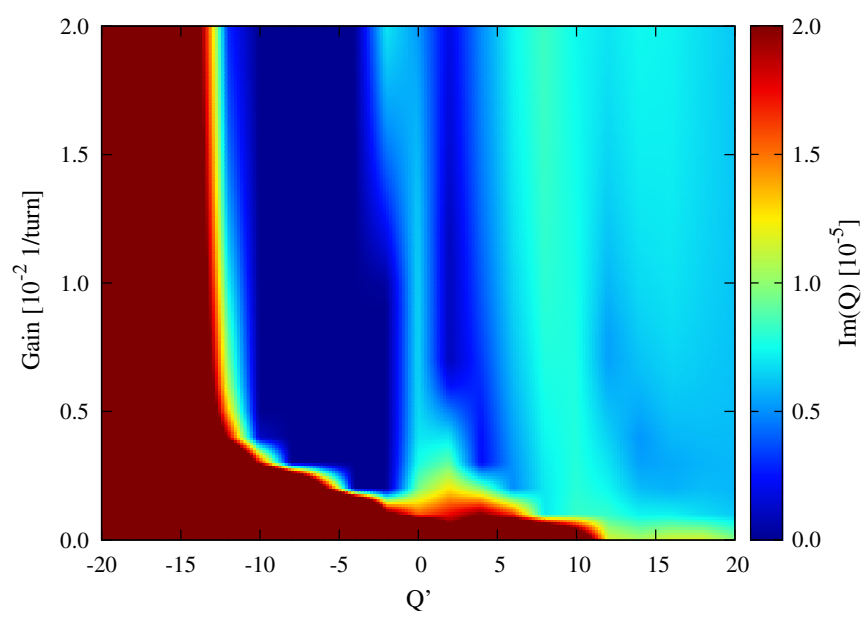

FIG. 19. Imaginary part of the tune of the most unstable mode obtained with the CMM for two trains of 16 bunches colliding in one IP, for different chromaticity and damper gain at separations of $13 \sigma$. 
derivations by means of stability diagrams. In addition, the presence of mode coupling instabilities drastically increases the difficulty to analytically evaluate the effect of Landau damping on the modes considered. Tracking simulations are therefore well suited for this kind of study. The results of tracking simulations with different chromaticity and transverse feedback settings are compared to the results obtained with the CMM in Fig. 18. The maximum tune shift is deduced from tracking simulations by performing a singular value decomposition of all bunch centroid positions, turn by turn, and fitting an exponential to the time evolution singular vector corresponding to the largest singular value. At $Q^{\prime}=0$, the coupled mode instability observed at $12 \sigma$ is in agreement with the CMM. The second peak at $9 \sigma$ separation is not visible, suggesting that it is Landau damped. More generally, discrepancies for all cases are observed at low separations for which the beam-beam tune spread becomes relevant.

Increasing the chromaticity to 10 significantly mitigates the mode coupling instability while a damper gain of 0.01 appears to fully suppress it. These observations are consistent with the single bunch results presented in Sec. V where it was shown that high chromaticity and damper gain can efficiently stabilize the mode coupling instabilities.

At 0 chromaticity and without transverse feedback, there is a significant discrepancy between the two models for separations larger than $14 \sigma$. This discrepancy may be attributed to the non-normality of the system [24]. In the absence of synchrotron motion, non-normal instabilities arise due to the fact that the wakefield affects only trailing particles. These are known as the beam breakup instabilities observed in linear accelerators [20]. While synchrotron motion restores the normality of the system when considering intrabunch motion, multibunch effects remain nonnormal when the multiturn wake is neglected, having strong consequences for both models. Within the CMM, it leads to nondiagonalizable matrices. As a consequence the eigenvectors are no longer fully representative of the dynamic of the system. Also, the behavior observed in tracking simulations is dominated by transient, i.e., nonexponential, growth, which are characteristic of non-normal systems [25]. The exponential fit of simulation data is therefore not appropriate to describe the beams stability. Nevertheless, the agreement between the two codes with high chromaticity and high transverse feedback gain suggests that the non-normal mechanisms are suppressed in these configurations, which is expected as single bunch mechanism becomes dominant over multibunch ones.

\section{B. Extrapolation to large number of bunches and LHC operational scenarios}

The LHC beams are composed of up to 2808 bunches. These bunches are coupled through the machine impedance and the multiple beam-beam interactions in the four interaction regions leading to a very complex system.
Both the CMM and tracking simulations become very demanding in term of computing resources with a large number of bunches and simulation of the full machine are, for now, out of reach.

Nevertheless, the case of a train of 16 bunches colliding in a single interaction region provides a better understanding of the behavior of the full machine under changes of critical parameters, such as beam-beam separation, chromaticity and damper gain. As shown by Fig. 20, while the largest imaginary tune shift is affected when changing the length of a bunch train from 16 to 64 bunches, the overall behavior as a function of the separation between the beams is similar in all configurations. It is important to note that we considered a machine configuration with 16 possible beam-beam interactions in a single interaction region, therefore the number of interactions per bunch is identical for all trains of more than 16 bunches. Also, the simulations presented include a single interaction region, whereas the LHC counts four in total placed asymmetrically around the ring. Accounting for the full complexity of the problem would require the presence of the maximum number of bunches, which as already mentioned is out of reach. Nevertheless, simulations with the CMM show a similar behavior with simplified, yet different configurations. While the absolute values of rise times and real tune shifts obtained vary, the characteristics of the coupled mode instability shown on Figs. 18 and 19 remained valid in the configurations tested.

Similarly to the single bunch case, both high damper gain and high chromaticity appear to mitigate the coupling between low order modes. At high damper gain, two regions of stability, with negative or particularly small imaginary tune shift, are observed. They appear in blue in Fig. 19 and are located between chromaticities of approximately 1.0 to
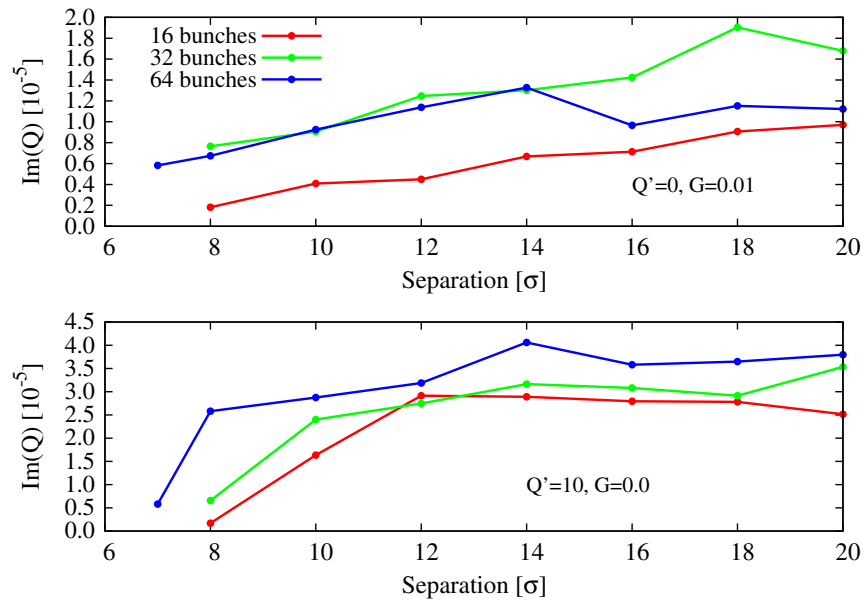

FIG. 20. Imaginary tune shift of the most unstable mode from tracking simulations with varying number of bunches. On the top plot, the chromaticity is 0 and transverse feedback gain is 0.01 , on the bottom plot, the chromaticity is 10 and the transverse feedback gain is 0 . 
5.0 and -2.0 to -10.0 . While appealing due to its large area, the stability region at negative chromaticity, with imaginary tune shifts exclusively below 0 was found to significantly shrink when the beam-beam tune shift increases. This feature makes it hardly usable for regular operation, as issues may arise when going to full head-on collisions. On the other hand, the slightly positive chromaticities remain rather stable even at large beam-beam tune shifts and would provide a usable area with minimal tune shifts for the whole LHC cycle. This stability band is, however, quite narrow and would require a control of the chromaticity on the unit level.

\section{SUMMARY}

We developed numerical tools, both analytical and multiparticle tracking, to study the interplay of beam-beam and impedance. These tools were fully benchmarked against each other and existing instability codes showing excellent agreement for all the cases tested.

Using these numerical tools, we demonstrated the existence of a strong mode coupling instability when one of the beam-beam coherent modes crossed a higher order headtail mode. This instability which has very similar characteristics to the classical impedance driven TMCI could occur even at low bunch intensities providing the beam-beam parameter is sufficiently large for the beambeam coherent modes frequency to reach higher order headtail modes. While chromaticity appears to be rather inefficient by itself to cure this instability, a bunch-bybunch transverse damper would easily suppress it. Extensive parameters scans showed that an optimum combination of damper gain and chromaticity could be found in order to minimize unwanted side effects related to high chromaticity or damper gain such as lifetime or emittance degradation.

A short experiment was performed with colliding beams with transverse offsets. A strong instability was observed in the absence of transverse damper at separation consistent with the mode coupling instability. The data are not fully conclusive and could only be partially reproduced in simulations due to large uncertainty on key parameters such as the impedance model or the chromaticity. Nevertheless, a comparison between weak-strong, featuring no coherent beam-beam modes, and strong-strong simulations showed that this strong instability should not occur in the absence of coherent beam-beam modes. This observation shows their importance in the process driving this instability and tends to confirm the hypothesis of the mode coupling instability involving coherent beam-beam modes.

Finally, we performed multibunch simulations as relevant for LHC operation confirming the results obtained with the reduced single bunch approximation, i.e., an appropriate combination of transverse damper and chromaticity should allow one to suppress these instabilities.

\section{ACKNOWLEDGMENTS}

The authors would like to thank J. Wenninger, S. Redaelli, and the LHC operation team for their support with the data acquisition. We would like to express special thanks to A. Valishev who suggested the circulant matrix approach and helped with its implementation as well as J. Qiang for sharing his code BEAMBEAM3D. Finally, we would like to thank E. Métral and the members of the CERN/ABP-ICE section for their support and valuable discussions on collective effects. This work is partially supported by Brookhaven Science Associates, LLC under Contract No. DE-AC02-98CH10886 and LARP with the U.S. Department of Energy. This research used resources of the National Energy Research Scientific Computing Center, which is supported by the Office of Science of the U.S. Department of Energy under Contract No. DEAC02-05CH11231. This research has used resources of the High Performance Computing center of the Ecole Polytechnique Federale de Lausanne, which was supported by the Laboratory of Particle Accelerators Physics.

[1] K. Yokoya and H. Koiso, Part. Accel. 27, 181 (1990).

[2] A. Piwinski, IEEE Trans. Nucl. Sci. 26, 4267 (1979).

[3] K. Hirata, Nucl. Instrum. Methods Phys. Res., Sect. A 269, 7 (1988).

[4] W. Fischer, M. Blaskiewicz, J. M. Brennan, P. Cameron, R. Connolly, C. Montag, S. G. Peggs, S. Tepikian, V. Ptitsyn, D. Trbojevic, and J. van Zeijts, in Proceedings of the 20th Particle Accelerator Conference, PAC-2003, Portland, OR, 2003 (IEEE, New York, 2003).

[5] G. Stancari and A. A. Valishev, Phys. Rev. ST Accel. Beams 15, 041002 (2012).

[6] Y. Alexahin, Nucl. Instrum. Methods Phys. Res., Sect. A 480, 253 (2002).

[7] E. A. Perevedentsev and A. A. Valishev, Phys. Rev. ST Accel. Beams 4, 024403 (2001).

[8] E. G. Stern, J. F. Amundson, P. G. Spentzouris, and A. A. Valishev, Phys. Rev. ST Accel. Beams 13, 024401 (2010).

[9] T. Pieloni, G. Arduini, X. Buffat, R. Giachino, W. Herr, M. Lamont, E. Metral, N. Mounet, G. Papotti, B. Salvant, J. Wenninger, and S. White, in Proceedings of the 4th International Particle Accelerator Conference, IPAC-2013 (JACoW, Shanghai, China, 2013).

[10] E. Métral, G. Arduini, D. Banfi, J. Barranco, H. Bartosik, X. Buffat, O. Bruning, S. Fartoukh, W. Hofle, W. Herr, G. Iadarola, M. Kuhn, K. Li, N. Mounet, T. Pieloni, G. Rumolo, B. Salvachua Ferrando, F. Zimmermann, A. Burov, and S. White, Report No. CERN-ACCNOTE-2014-0006, 2014.

[11] V. V. Danilov and E. A. Perevedenstsev, Nucl. Instrum. Methods Phys. Res., Sect. A 391, 77 (1997).

[12] J. Qiang, M. A. Furman, R. D. Ryne, W. Fischer, and K. Ohmi, Nucl. Instrum. Methods Phys. Res., Sect. A 558, 351 (2006).

[13] T. Pieloni, Ph.D. thesis, EPFL, 2008. 
[14] W. Herr, M. P. Zorzano, and F. Jones, Report No. CERNLHC466, 2001.

[15] G. Rumolo and F. Zimmermann, Phys. Rev. ST Accel. Beams 5, 121002 (2002).

[16] N. Mounet, Ph.D. thesis, EPFL, 2012.

[17] B. Salvachua, R. W. Assmann, R. Bruce, M. Cauchi, D. Deboy, L. Lari, A. Marsili, D. Mirarchi, E. Quaranta, S. Redaelli, A. Rossi, and G. Valentino, LHC Beam Operation Workshop Proceedings (2012).

[18] O. S. Bruning, P. Collier, P. Lebrun, S. Myers, R. Ostojic, J. Poole, and P. Proudlock, Report No. CERN-2004-003-V-1, 101, 2004.

[19] N. Mounet, X. Buffat, R. Bruce, W. Herr, E. Métral, G. Rumolo, and B. Salvant, LHC Beam Operation Workshop Proceedings (2011).
[20] A. Chao, Physics of Collective Instabilities in High Energy Accelerators (Wiley, New York, 1993).

[21] Y. Alexahin, in Proceedings of the 21st Particle Accelerator Conference, Knoxville, TN, 2005 (IEEE, Piscataway, NJ, 2005).

[22] B. Muratori and T. Pieloni, ICFA BB2013 Workshop Proceedings (2013).

[23] X. Buffat, ICFA BB2013 Workshop Proceedings (2013).

[24] L. N. Trefethen and M. Embree, Spectra and Pseudospectra: The Behavior of Nonnormal Matrices and Operators (Princeton University Press, Princeton, NJ, 2005).

[25] P. J. Schmid, Annu. Rev. Fluid Mech. 39, 129 (2007). 